Napoli - DSF - $99-11$

\title{
Leptonic and Semileptonic Decays of Pseudoscalar Mesons
}

\author{
M.A. Ivanov ${ }^{a}$ and P. Santorelli ${ }^{b}$ \\ ${ }^{a}$ Bogoliubov Laboratory of Theoretical Physics, \\ Joint Institute for Nuclear Research, 141980 Dubna, Russia \\ b Dipartimento di Scienze Fisiche, \\ Università "Federico II" di Napoli, Napoli, Italy \\ and \\ INFN Sezione di Napoli
}

\begin{abstract}
We employ the relativistic constituent quark model to give a unified description of the leptonic and semileptonic decays of pseudoscalar mesons $\left(\pi, K, D, D_{s}, B, B_{s}\right)$. The calculated leptonic decay constants and form factors are found to be in good agreement with available experimental data and other approaches. We reproduce the results of spin-flavor symmetry in the heavy quark limit.
\end{abstract}

\section{Introduction}

Semileptonic decays of pseudoscalar mesons allow to evaluate the elements of the Cabibbo-Kobayashi-Maskawa (CKM) matrix, which are fundamental parameters of the Standard Model. The decay $K \rightarrow \pi e \nu$ provides the most accurate determination of $V_{u s}$, the semileptonic decays of $\mathrm{D}$ and $\mathrm{B}$ mesons, $D \rightarrow K\left(K^{*}\right) l \nu, B \rightarrow D\left(D^{*}\right) l \nu$ and $B \rightarrow \pi(\rho) l \nu$, can be used to determine $\left|V_{c s}\right|,\left|V_{c b}\right|$ and $\left|V_{u b}\right|$, respectively. The effects of strong interactions in these processes can be expressed in terms of form factors, which depend on $q^{2}$, the squared momentum transferred to the leptonic pair. Information on the form factors are obtained by measuring the distributions of $q^{2}$ and decay angles.

The decays of heavy D and B mesons are of particular interest due to the spinflavor symmetry observed for infinite quark masses [1]. This symmetry allows 
to reduce the number of form factors and express them in terms of the universal Isgur-Wise function [2]. Also the scaling laws derived for some physical observables can be, in principle, tested experimentally. Since the Isgur-Wise function cannot be calculated from first principles, many models and nonperturbative approaches, which exhibit the heavy quark symmetry, have been employed to describe relevant phenomena. However, it was found out, that the finite mass corrections are very important, especially, in the charm sector. It appears that in some sense a step back should be done from using the heavy quark symmetry as a guide under model building to the straightforward calculations with full quark propagators. Then one has to check the consistency of the results with the spin-flavor symmetry in the heavy quark limit.

In this paper we employ the relativistic constituent quark model (RCQM) [3] for the simultaneous description of both light and heavy flavored meson leptonic and semileptonic decays. This model is based on the effective Lagrangian describing the coupling of mesons with their quark constituents, and the compositeness condition. The physical processes are described by the one-loop quark diagrams with free constituent propagators and meson-quark vertices related to the Bethe-Salpeter amplitudes. The masses of lower-lying pseudoscalar (PS) mesons should be less than the sum of quark constituent masses to provide the absence of imaginary parts corresponding to quark production. The adjustable parameters, the widths of Bethe-Salpeter amplitudes in momentum space and constituent quark masses, are determined from the best fit of available experimental data and some lattice determinations. We found that our results are in good agreement with experimental data and other approaches. Also we reproduce the results of spin-flavor symmetry for leptonic decay constants and semileptonic form factors in the heavy quark limit.

The shapes of vertex functions and quark propagators should be found from the Bethe-Salpeter and Dyson-Schwinger equations, respectively. This is provided by the Dyson-Schwinger Equation (DSE) [4] studies. A DSE-approach has been employed to provide a unified and uniformly accurate description of light- and heavy-meson observables $[5,6]$.

A similar approach, based on the effective heavy meson Lagrangian, has been described in [7] in terms of a model based on meson-quark interactions, where mesonic transition amplitudes are represented by diagrams with heavy mesons attached to quark loops. The free propagator has been used for light quarks. However, the quark propagator obtained in the heavy quark limit has been employed for heavy quarks. 


\section{The model}

We employ an approach [3] based on the effective interaction Lagrangian which describes the transition of hadron into quarks. For example, the transition of the meson $H$ into its constituents $q_{1}$ and $q_{2}$ is given by the Lagrangian

$$
\mathcal{L}_{\text {int }}(x)=g_{H} H(x) \int d x_{1} \int d x_{2} \Phi_{H}\left(x ; x_{1}, x_{2}\right) \bar{q}\left(x_{1}\right) \Gamma_{H} \lambda_{H} q\left(x_{2}\right) .
$$

Here, $\lambda_{H}$ and $\Gamma_{H}$ are the Gell-Mann and Dirac matrices, respectively, which provide the flavor and spin numbers of the meson $H$. The function $\Phi_{H}$ is related to the scalar part of Bethe-Salpeter amplitude. For instance, the separable form $\Phi_{H}\left(x ; x_{1}, x_{2}\right)=\delta\left(x-\left(x_{1}+x_{2}\right) / 2\right) f\left(\left(x_{1}-x_{2}\right)^{2}\right)$ has been used in [3] for pions.

The coupling constants $g_{H}$ is given by the so called compositeness condition proposed in [8] and extensively used in [9]. That condition means that the renormalization constant of the meson field is equal to zero:

$$
Z_{H}=1-\frac{3 g_{H}^{2}}{4 \pi^{2}} \tilde{\Pi}_{H}^{\prime}\left(m_{H}^{2}\right)=0
$$

where $\tilde{\Pi}_{H}^{\prime}$ is the derivative of the meson mass operator defined by

$$
\tilde{\Pi}_{H}\left(p^{2}\right)=\int \frac{d^{4} k}{4 \pi^{2} i} \phi_{H}^{2}\left(-k^{2}\right) \operatorname{tr}\left[\Gamma_{H} S_{2}(\not k) \Gamma_{H} S_{1}(\not k+\not p)\right] .
$$

The invariant amplitudes describing the leptonic $H(p) \rightarrow l \nu$ and semileptonic $H(p) \rightarrow H^{\prime}\left(p^{\prime}\right) l \nu$ decays are written down

$$
\begin{aligned}
A(H(p) \rightarrow e \nu) & =\frac{G_{F}}{\sqrt{2}} V_{q q^{\prime}}\left(\bar{e} O_{\mu} \nu\right) M_{H}^{\mu}(p) \\
A\left(H(p) \rightarrow H^{\prime}\left(p^{\prime}\right) e \nu\right) & =\frac{G_{F}}{\sqrt{2}} V_{q q^{\prime}}\left(\bar{e} O_{\mu} \nu\right) M_{H H^{\prime}}^{\mu}\left(p, p^{\prime}\right),
\end{aligned}
$$

where $G_{F}$ is the Fermi weak-decay constant, $V_{q q^{\prime}}$ is the appropriate element of the CKM matrix. The matrix elements of the hadronic currents are given by

$$
M_{H}^{\mu}(p)=\frac{3}{4 \pi^{2}} g_{H} \int \frac{d^{4} k}{4 \pi^{2} i} \phi_{H}\left(-k^{2}\right) \operatorname{tr}\left[\gamma^{5} S_{2}(\not k) O^{\mu} S_{1}(\not k+\not p)\right]=f_{H} p^{\mu}
$$




$$
\begin{aligned}
M_{H H^{\prime}}^{\mu}\left(p, p^{\prime}\right)= & \frac{3}{4 \pi^{2}} g_{H} g_{H^{\prime}} \int \frac{d^{4} k}{4 \pi^{2} i} \phi_{H}\left(-k^{2}\right) \phi_{H^{\prime}}\left(-k^{2}\right) \\
& \times \operatorname{tr}\left[\gamma^{5} S_{3}(\not k) \gamma^{5} S_{2}\left(\not k+\not p^{\prime}\right) O^{\mu} S_{1}(\not k+\not p)\right] \\
& =f_{+}\left(q^{2}\right)\left(p+p^{\prime}\right)^{\mu}+f_{-}\left(q^{2}\right)\left(p-p^{\prime}\right)^{\mu}
\end{aligned}
$$

where $\phi_{H}\left(-k^{2}\right)$ is related to the BS-amplitude in momentum space, and

$$
S_{i}(\not k)=\frac{1}{m_{i}-\not k}
$$

is the propagator of the constituent quark with mass $m_{i}$. As discussed before, to avoid the appearance of imaginary parts in Eqs. (6) and (7), we assume that $m_{H}<m_{q_{1}}+m_{q_{2}}$ which is a reliable approximation for the lower-lying mesons considered here.

To evaluate the integral in Eq. (7)

$$
I_{H H^{\prime}}\left(p, p^{\prime}\right)=\int \frac{d^{4} k}{4 \pi^{2} i} \mathcal{F}\left(-k^{2}\right) \operatorname{tr}\left\{\gamma^{5} S_{3}(\not k) \gamma^{5} S_{2}\left(\not k+\not p^{\prime}\right) \gamma^{\mu} S_{1}(\not k+\not p)\right\},
$$

where $\mathcal{F}\left(-k^{2}\right)=\phi_{H}\left(-k^{2}\right) \cdot \phi_{H^{\prime}}\left(-k^{2}\right)$, we need to calculate the following integrals:

$$
J^{(0, \mu, \mu \nu, \mu \nu \delta)}=\int \frac{d^{4} k}{\pi^{2} i} \frac{\left(1, k^{\mu}, k^{\mu} k^{\nu}, k^{\mu} k^{\nu} k^{\delta}\right) \mathcal{F}\left(-k^{2}\right)}{\left[m_{1}^{2}-(k+p)^{2}\right]\left[m_{2}^{2}-\left(k+p^{\prime}\right)^{2}\right]\left[m_{3}^{2}-k^{2}\right]}
$$

Using the Cauchy representation for the function $\mathcal{F}\left(-k^{2}\right)$ and then the standard techniques of the Feynman $\alpha$-parametrization one finds $\left(\mathcal{F}^{\prime}(z)=d \mathcal{F}(z) / d z\right)$

$$
\begin{aligned}
J^{0} & =\int_{0}^{\infty} d t\left(\frac{t}{1+t}\right)^{2} \int d^{3} \alpha \delta\left(1-\sum_{i=1}^{3} \alpha_{i}\right)\left(-\mathcal{F}^{\prime}\left(z_{I}\right)\right) \\
J^{\mu} & =-\int_{0}^{\infty} d t\left(\frac{t}{1+t}\right)^{3} \int d^{3} \alpha \delta\left(1-\sum_{i=1}^{3} \alpha_{i}\right) P_{\alpha}^{\mu}\left(-\mathcal{F}^{\prime}\left(z_{I}\right)\right) \\
J^{\mu \nu} & =\int_{0}^{\infty} d t\left(\frac{t}{1+t}\right)^{2} \int d^{3} \alpha \delta\left(1-\sum_{i=1}^{3} \alpha_{i}\right)
\end{aligned}
$$




$$
\begin{aligned}
& \times\left\{-\frac{1}{2} g^{\mu \nu} \frac{1}{1+t} \mathcal{F}\left(z_{I}\right)-P_{\alpha}^{\mu} P_{\alpha}^{\nu}\left(\frac{t}{1+t}\right)^{2} \mathcal{F}^{\prime}\left(z_{I}\right)\right\} \\
J^{\mu \nu \delta}= & \int_{0}^{\infty} d t\left(\frac{t}{1+t}\right)^{2} \int d^{3} \alpha \delta\left(1-\sum_{i=1}^{3} \alpha_{i}\right) \\
& \times\left\{\frac{1}{2}\left[g^{\mu \nu} P_{\alpha}^{\delta}+g^{\mu \delta} P_{\alpha}^{\nu}+g^{\nu \delta} P_{\alpha}^{\mu}\right] \frac{t}{(1+t)^{2}} \mathcal{F}\left(z_{I}\right)\right. \\
& \left.+P_{\alpha}^{\mu} P_{\alpha}^{\nu} P_{\alpha}^{\delta}\left(\frac{t}{1+t}\right)^{3} \mathcal{F}^{\prime}\left(z_{I}\right)\right\}
\end{aligned}
$$

where $q=p-p^{\prime}, P_{\alpha}=\alpha_{1} p+\alpha_{2} p^{\prime}, D_{3}=\alpha_{1} \alpha_{3} p^{2}+\alpha_{2} \alpha_{3} p^{\prime 2}+\alpha_{1} \alpha_{2} q^{2}$, and $z_{I}=t\left[\sum_{i=1}^{3} \alpha_{i} m_{i}^{2}-D_{3}\right]-P_{\alpha}^{2} t /(1+t)$.

Finally, Eq. (10) becomes

$$
I_{H H^{\prime}}^{\mu}\left(p, p^{\prime}\right)=\left(p+p^{\prime}\right)^{\mu} I_{+}\left(p^{2}, p^{\prime 2}, q^{2}\right)+\left(p-p^{\prime}\right)^{\mu} I_{-}\left(p^{2}, p^{\prime 2}, q^{2}\right)
$$

with

$$
\begin{aligned}
I_{+}\left(p^{2}, p^{\prime 2}, q^{2}\right)= & \frac{1}{2} \int_{0}^{\infty} d t\left(\frac{t}{1+t}\right)^{2} \int d^{3} \alpha \delta\left(1-\sum_{i=1}^{3} \alpha_{i}\right) \\
\times & \left\{\mathcal{F}\left(z_{I}\right) \frac{1}{1+t}\left[4-3\left(\alpha_{1}+\alpha_{2}\right) \frac{t}{1+t}\right]\right. \\
& -\mathcal{F}^{\prime}\left(z_{I}\right)\left[\left(m_{1}+m_{2}\right) m_{3}\right. \\
& +\frac{t}{1+t}\left(-\left(\alpha_{1}+\alpha_{2}\right)\left(m_{1} m_{3}+m_{2} m_{3}-m_{1} m_{2}\right)\right. \\
& \left.\left.\left.+\alpha_{1} p^{2}+\alpha_{2} p^{\prime 2}\right)-P_{\alpha}^{2}\left(\frac{t}{1+t}\right)^{2}\left(2-\left(\alpha_{1}+\alpha_{2}\right) \frac{t}{1+t}\right)\right]\right\} .
\end{aligned}
$$

The normalization condition is written in the form

$$
\frac{3 g_{H}^{2}}{4 \pi^{2}} I_{+}\left(p^{2}, p^{2}, 0\right)=1
$$

with $m_{1}=m_{2} \equiv m$.

The integrals corresponding to the matrix element of the leptonic decay $H(p) \rightarrow$ $l \nu$ and radiative decay of neutral meson $H(p) \rightarrow \gamma\left(q_{1}\right)+\gamma\left(q_{2}\right)$ are calculated following the same procedure. We have 


$$
\begin{aligned}
Y^{\mu}(p) & =\int_{0} \frac{d^{4} k}{4 \pi^{2} i} \phi\left(-k^{2}\right) \operatorname{tr}\left\{\gamma^{5} S_{2}(\not k) \gamma^{\mu}\left(I-\gamma^{5}\right) S_{1}(\not k+\not p)\right\}=p^{\mu} Y\left(p^{2}\right) \\
Y\left(p^{2}\right) & =\int_{0}^{\infty} d t \frac{t}{(1+t)^{2}} \int_{0}^{1} d \alpha\left[m_{2}+\left(m_{1}-m_{2}\right) \frac{\alpha t}{1+t}\right] \phi\left(z_{Y}\right) \\
K^{\mu \nu}\left(q_{1}, q_{2}\right) & =\int \frac{d^{4} k}{4 \pi^{2} i} \phi\left(-k^{2}\right) \operatorname{tr}\left\{\gamma^{5} S\left(\not k-\not q_{2}\right) \gamma^{\mu} S(\not k) \gamma^{\nu} S\left(\not k+\not q_{1}\right)\right\} \\
& =i \varepsilon^{\mu \nu \alpha \beta} q_{1}^{\alpha} q_{2}^{\beta} K\left(p^{2}\right) \\
K\left(p^{2}\right) & =m \int_{0}^{\infty} d t\left(\frac{t}{1+t}\right)^{2} \int_{0}^{1} d \alpha_{1} \int_{0}^{1-\alpha_{1}} d \alpha_{2}\left(-\phi^{\prime}\left(z_{K}\right)\right)
\end{aligned}
$$

where $z_{Y}=t\left[\alpha m_{1}^{2}+(1-\alpha) m_{2}^{2}-\alpha p^{2}+\alpha^{2} p^{2} t /(1+t)\right]$ and $z_{K}=t\left[m_{1}^{2}-\alpha_{1} \alpha_{2} p^{2}\right]+$ $\alpha_{1} \alpha_{2} p^{2} t /(1+t)$.

The physical observables are expressed in terms of the structural integrals written in Eqs. (16), (18) and (19):

$$
\begin{gathered}
g_{P \gamma \gamma}=\frac{g_{P}}{2 \sqrt{2} \pi^{2}} K\left(m_{P}^{2}\right), \quad \Gamma(P \rightarrow \gamma \gamma)=\frac{\pi}{4} \alpha^{2} m_{P}^{3} g_{P \gamma \gamma}^{2}, \\
f_{P}=\frac{3}{4 \pi^{2}} g_{P} Y\left(m_{P}^{2}\right), \quad \Gamma(P \rightarrow l \nu)=\left|V_{q q^{\prime}}\right|^{2} \frac{G_{F}^{2} f_{P}^{2}}{8 \pi} m_{P} m_{l}^{2}\left[1-\frac{m_{l}^{2}}{m_{P}^{2}}\right]^{2},(21) \\
f_{+}\left(q^{2}\right)=\frac{3}{4 \pi^{2}} g_{P} g_{P^{\prime}} I_{+}\left(m_{P}^{2}, m_{P^{\prime}}^{2}, q^{2}\right), \\
\Gamma\left(P \rightarrow P^{\prime} l \nu\right)=\left|V_{q q^{\prime}}\right|^{2} \frac{G_{F}^{2}}{192 \pi^{3} m_{P}^{3}} \int_{0}^{t_{-}} d t\left|f_{+}(t)\right|^{2}\left[\left(t_{+}-t\right)\left(t_{-}-t\right)\right]^{3 / 2},
\end{gathered}
$$

with $t_{ \pm}=\left(m_{P} \pm m_{P^{\prime}}\right)^{2}$ (the extra factor $1 / 2$ appears for $\pi^{0}$ in the final state).

\subsection{Heavy quark limit}

The leptonic heavy decay constants and semileptonic heavy to heavy form factors acquire a simple form in the heavy quark limit, $i$. e. when $m_{1} \equiv M \rightarrow$ $\infty, m_{2} \equiv M^{\prime} \rightarrow \infty$ and $p^{2}=(M+E)^{2}, p^{\prime 2}=\left(M^{\prime}+E\right)^{2}$ with $E$ being a constant value. From Eq. (16) by replacing the variables $\alpha_{1} \rightarrow \alpha_{1} / M$ and $\alpha_{2} \rightarrow \alpha_{2} / M^{\prime}$, one obtains 


$$
\begin{aligned}
I_{+} & \rightarrow \frac{M+M^{\prime}}{2 M M^{\prime}} \cdot \int_{0}^{\infty} d t\left(\frac{t}{1+t}\right)^{2} \int_{0}^{1} d \alpha \alpha \int_{0}^{1} d \tau\left(-\mathcal{F}^{\prime}(z)\right)\left[m+\frac{\alpha t}{1+t}\right] \\
& =\frac{M+M^{\prime}}{2 M M^{\prime}} \cdot \frac{1}{2} \int_{0}^{1} \frac{d \tau}{W} \int_{0}^{\infty} d u \mathcal{F}(\tilde{z}) \frac{m+\sqrt{u}}{m^{2}+\tilde{z}}
\end{aligned}
$$

where $\tilde{z}=u-2 E \sqrt{u / W}, W=1+2 \tau(1-\tau)(w-1)$ and $w=\left(M^{2}+M^{\prime 2}-\right.$ $\left.2 M M^{\prime} q^{2}\right) /\left(2 M M^{\prime}\right)$.

The normalization condition can be obtained from Eq. (23) by putting $w=1$ and $M^{\prime}=M$. We have

$$
\frac{3 g_{H}^{2}}{4 \pi^{2}} \cdot I_{+}^{(0)}=1, \quad I_{+}^{(0)}=\frac{1}{2 M} I_{N}, \quad I_{N}=\int_{0}^{\infty} d u \phi_{H}^{2}\left(\tilde{z}_{0}\right) \frac{m+\sqrt{u}}{m^{2}+\tilde{z}_{0}}
$$

where $\tilde{z}_{0}=u-2 E \sqrt{u}$. Then the leptonic decay constant and semileptonic form factors are written as

$$
\begin{aligned}
& f_{P} \rightarrow \frac{1}{\sqrt{M}} \cdot \sqrt{\frac{3}{2 \pi^{2} I_{N}}} \int_{0}^{\infty} d u[\sqrt{u}-E] \phi_{H}\left(\tilde{z}_{0}\right) \frac{m+\sqrt{u} / 2}{m^{2}+\tilde{z}_{0}} \\
& f_{ \pm} \rightarrow \frac{M^{\prime} \pm M}{2 \sqrt{M M^{\prime}}} \xi(w) \quad \xi(w)=\frac{1}{I_{N}} \int_{0}^{1} \frac{d \tau}{W} \int_{0}^{\infty} d u \phi_{H}^{2}(\tilde{z}) \frac{m+\sqrt{u}}{m^{2}+\tilde{z}} .
\end{aligned}
$$

It is readily seen that we reproduce the scaling law for both leptonic decay constants and form factors, and obtain the explicit expression for the IsgurWise function $[1,2]$.

\section{Results and discussion}

The expressions obtained in the previous section for physical observables are valid for any vertex function $\phi_{H}\left(-k^{2}\right)$. Here, we choose a Gaussian form $\phi\left(-k^{2}\right)=\exp \left\{k^{2} / \Lambda_{H}^{2}\right\}$ in Minkowski space. The magnitude of $\Lambda_{H}$ characterizes the size of the BS-amplitude and is an adjustable parameter in our approach. Thus, we have six $\Lambda$-parameters plus the four quark masses, all of which are fixed via the least-squares fit to the observables measured experimentally or taken from lattice simulations (see, Table 1).

The fit yields the values of $\Lambda$-parameters and the constituent quark masses 
Table 1

Calculated values of a range of observables $\left(g_{\pi \gamma \gamma}\right.$ in $\mathrm{GeV}^{-1}$, leptonic decay constants in $\mathrm{GeV}$, form factors and ratios are dimensionless). The "Obs." are extracted from Refs. [10-16]. The quantities used in fitting our parameters are marked by “*”.

\begin{tabular}{llll|lll} 
& & Obs. & Calc. & & Obs. & Calc. \\
\hline$*$ & $g_{\pi \gamma \gamma}$ & 0.274 & 0.242 & $f_{+}^{K \pi}(0)$ & 0.98 & 0.98 \\
$*$ & $f_{\pi}$ & 0.131 & 0.131 & $* f_{+}^{D K}(0)$ & $0.74 \pm 0.03$ & 0.74 \\
$*$ & $f_{K}$ & 0.160 & 0.160 & $f_{+}^{B D}(0)$ & & 0.73 \\
$*$ & $f_{D}$ & $0.191_{-28}^{+19}$ & 0.191 & $f_{+}^{B \pi}(0)$ & $0.27 \pm 0.11$ & 0.51 \\
$*$ & $\frac{f_{D_{s}}}{f_{D}}$ & $1.08(8)$ & 1.08 & $\operatorname{Br}(K \rightarrow \pi l \nu)$ & $(4.82 \pm 0.06) \cdot 10^{-2}$ & $4.4 \cdot 10^{-2}$ \\
& $f_{D_{s}}$ & $0.206_{-28}^{+18}$ & 0.206 & $\operatorname{Br}(D \rightarrow K l \nu)$ & $(6.8 \pm 0.8) \cdot 10^{-2}$ & $8.1 \cdot 10^{-2}$ \\
$*$ & $f_{B}$ & $0.172_{-31}^{+27}$ & 0.172 & $\operatorname{Br}(B \rightarrow D l \nu)$ & $(2.00 \pm 0.25) \cdot 10^{-2}$ & $2.3 \cdot 10^{-2}$ \\
$*$ & $\frac{f_{B_{s}}}{f_{B}}$ & $1.14(8)$ & 1.14 & $\operatorname{Br}(B \rightarrow \pi l \nu)$ & $(1.8 \pm 0.6) \cdot 10^{-4}$ & $2.1 \cdot 10^{-4}$ \\
& $f_{B_{s}}$ & & 0.196 & & & \\
\hline
\end{tabular}

which are listed in Eqs. (27) and (28).

\begin{tabular}{|c|c|c|c|c|c|}
\hline$\Lambda_{\pi}$ & $\Lambda_{K}$ & $\Lambda_{D}$ & $\Lambda_{D_{s}}$ & $\Lambda_{B}$ & (in $\mathrm{GeV}$ ) \\
\hline \multirow[t]{3}{*}{1.16} & 1.82 & 1.87 & 1.95 & 2.16 & 2.27 \\
\hline & $m_{u}$ & $m_{s}$ & $m_{c}$ & $m_{b}$ & (in $\mathrm{GeV}$ ) \\
\hline & 0.235 & 0.333 & 1.67 & 5.06 & \\
\hline
\end{tabular}

The values of $\Lambda$ are such that $\Lambda_{m_{i}}<\Lambda_{m_{j}}$ if $m_{i}<m_{j}$. This corresponds to the ordering law for sizes of bound states. The values of $\Lambda_{D}=1.87 \mathrm{GeV}$ and $\Lambda_{B}=2.16 \mathrm{GeV}$ are larger than those obtained in [6]: $\Lambda_{D}=1.41 \mathrm{GeV}$ and $\Lambda_{B}=1.65 \mathrm{GeV}$. The mass of u-quark and the parameter $\Lambda_{\pi}$ are almost fixed from the decays $\pi \rightarrow \mu \nu$ and $\pi^{0} \rightarrow \gamma \gamma$ with an accuracy of a few percent. The obtained value of the u-quark mass $m_{u}=0.235 \mathrm{GeV}$ is less than the constituent-light-quark mass typically employed in quark models for baryon physics $\left(m_{u}>m_{N} / 3=0.313 \mathrm{GeV}\right)$. For instance, the value of $m_{u}=0.420$ $\mathrm{GeV}$ was extracted from fitting nucleon observables within our approach [3]. The different choice of constituent quark masses is a common feature of quark models with free propagators due to the lack of confinement. However, we consider here the low-lying mesons that allows us to fix the constituent quark masses in a self-consistent manner. As mentioned above, the meson masses must be less than the sum of masses of their constituents. This gives the restrictions on the choice of the meson binding energies: $E_{K}=m_{K}-m_{s}<m_{u}$, $E_{D}=m_{D}-m_{c}<m_{u}$ and $E_{B}=m_{B}-m_{b}<m_{u}$, which means that the 
binding energy cannot be relatively large as compared with those obtained in [6]: $E_{D}=0.58 \mathrm{GeV}$ and $E_{B}=0.74 \mathrm{GeV}$.

Let us now consider the $q^{2}$-behaviour of the form factors. We use the threeparameter function for the four $f_{+}$form factors

$$
f_{+}^{H H^{\prime}}\left(q^{2}\right)=\frac{f(0)}{1-b_{0}\left(q^{2} / m_{H}^{2}\right)-b_{1}\left(q^{2} / m_{H}^{2}\right)^{2}}
$$

here $b_{0}, b_{1}$ and $f(0)$ are parameters to be fitted. We collect the fitted values in the following Table and report the $q^{2}$-dependence in Fig. 1.

\begin{tabular}{c|cccc} 
& $K \rightarrow \pi$ & $D \rightarrow K$ & $B \rightarrow D$ & $B \rightarrow \pi$ \\
\hline$b_{0}$ & 0.28 & 0.64 & 0.77 & 0.52 \\
$b_{1}$ & 0.057 & 0.20 & 0.19 & 0.38
\end{tabular}

For comparison, we plot, together with our results, the predictions of a vector dominance monopole model:

$$
f_{+}^{q \rightarrow q^{\prime}}\left(q^{2}\right)=\frac{f_{+}^{q \rightarrow q^{\prime}}(0)}{1-q^{2} / m_{V_{q q^{\prime}}}^{2}}
$$

with $m_{V_{q q^{\prime}}}^{2}$ being a mass of lower-lying $\bar{q} q^{\prime}$-vector meson. We choose $m_{D_{s}^{*}}=2.11$ $\mathrm{GeV}$ for $c \rightarrow s, m_{B^{*}}=5.325 \mathrm{GeV}$ for $b \rightarrow u, m_{B_{c}^{*}} \approx m_{B_{c}}=6.4 \mathrm{GeV}$ [17] for $b \rightarrow c$ transitions. The values of $f_{+}^{q q^{\prime}}(0)$ are taken from the Table 1 . Also we calculate the branching ratios of semileptonic decays by using widely accepted values of the CKM matrix elements [10].

Our result for the slope of the $K_{l 3}$ form factor

$$
\lambda_{+}=m_{\pi}^{2} \frac{f_{+}^{K \pi \prime}(0)}{f_{+}^{K \pi}(0)}=0.023
$$

is in good agreement with experiment: $\lambda_{+}^{\text {expt }}=0.0286 \pm 0.0022$ [10] and VDM prediction: $\lambda_{+}^{\mathrm{VDM}}=m_{\pi}^{2} / m_{K^{*}}^{2}=0.025$. This value is also consistent with Refs. [18]

One can see that the agreement with experimental data and lattice results is very good, with the exception of the value of $f_{+}^{b u}(0)$ which is found to be larger than the monopole extrapolation of a lattice simulation, QCD Sum Rules (cf. [19]) and some other quark models (see, for example, [20,21]). However, this 
result is consistent with the value calculated from Refs. [6,22] and allows us to reproduce the experimental data for $B \rightarrow \pi l \nu$ with quite good accuracy.

\section{Acknowledgments}

We appreciate F. Buccella, V.E. Lyubovitskij, G. Nardulli and C.D. Roberts for many interesting discussions and critical remarks. We thank G. Esposito for reading the manuscript. M.A.I. gratefully acknowledges the hospitality and support of the Theory Group at Naples University where this work was conducted. This work was supported in part by the Russian Fund for Fundamental Research, under contract number 99-02-17731-a.

\section{References}

[1] N. Isgur and M.B. Wise, Phys. Lett. B232, 113 (1989); B237, 527 (1990).

[2] A.F. Falk, H. Georgi, B. Grinstein, M.B. Wise, Nucl. Phys. B343, 1 (1990).

[3] M.A. Ivanov and V.E. Lyubovitskij, Phys. Lett. B408, 435 (1997);

M.A. Ivanov, M.P. Locher and V.E. Lyubovitskij, Few-Body Syst. 21, 131 (1996).

[4] C.D. Roberts and A.G. Williams, Prog. Part. Nucl. Phys. 33, 477 (1994).

[5] M.A. Ivanov, Yu.L. Kalinovsky, P. Maris and C.D. Roberts, Phys. Rev. C57, 1991 (1998); Phys. Lett. B416, 29 (1998).

[6] M.A. Ivanov, Yu.L. Kalinovsky and C.D. Roberts, "Survey of heavy meson observables", nucl-th/9812063 (to appear in Phys. Rev. D).

[7] A. Deandrea, N. Di Bartolomeo, R. Gatto, G. Nardulli and A.D. Polosa, Phys. Rev. D58, 034004 (1998).

[8] A. Salam, Nuovo Cim. 25, 224 (1962);

S. Weinberg, Phys. Rev. 130, 776 (1963).

[9] G.V. Efimov and M.A. Ivanov, "The Quark Confinement Model of Hadrons", IOP Publishing, Bristol \& Philadelphia, 1993.

[10] Review of Particle Physics, (C. Caso et al.), Eur. Phys. J. C3, 1 (1998).

[11] CLEO Coll. (J. Bartelt et al.), "Measurement of the $B \rightarrow D l \nu$ branching fractions and form factors", hep-ex/9811042.

[12] CLEO Coll. (J. P. Alexander et al.), Phys. Rev. Lett. 77, 5000 (1996). 
[13] J.M. Flynn and C.T. Sachrajda, "Heavy Quark Physics from Lattice QCD", hep-lat/9710057.

[14] H. Wittig, Int. J. Mod. Phys. A12, 4477 (1997).

[15] UKQCD Coll. (L. Del Debbio et al.), Phys. Lett. B416, 392 (1998).

[16] MILC Coll. (C. Bernard et al.), Phys. Rev. Lett. 81, 4812 (1998).

[17] CDF Coll. (F. Abe et al.), Phys. Rev. D58, 112004 (1998).

[18] A. Afanasev and W.W. Buck, Phys. Rev. D55, 4380 (1997);

Yu. Kalinovsky, K.L. Mitchell and C.D. Roberts, Phys. Lett. B399, 22 (1997).

[19] P. Colangelo and P. Santorelli, Phys. Lett. B327, 123 (1994); V.M. Belyaev, V.M. Braun, A. Khodjamirian and R. Rückl, Phys. Rev. D51, 6177 (1995).

[20] M. Bauer, B. Stech and M. Wirbel, Z. Phys. C29,637 (1985); C34, 103 (1987); C42, 671 (1989).

[21] M. Ladisa, G. Nardulli, P. Santorelli, "Semileptonic and rare B meson decays into a light pseudoscalar meson", hep-ph/9903206.

[22] M.A. Ivanov and Yu.M. Valit, Mod. Phys. Lett. A12, 653 (1997).

[23] UKQCD Coll. (D.R. Burford et al.), Nucl. Phys. B447, 425 (1995). 

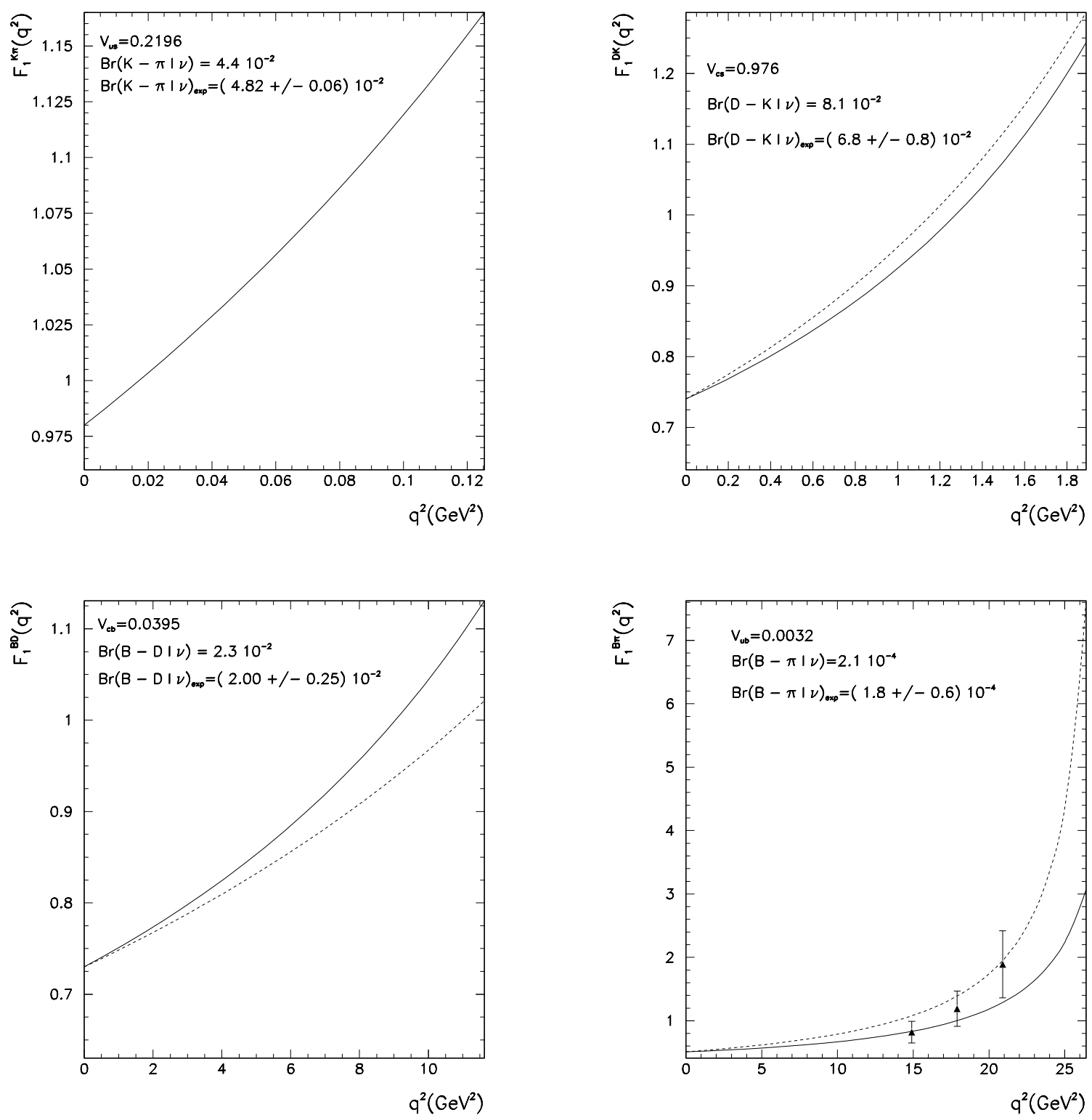

Fig. 1. The semileptonic $K \rightarrow \pi, D \rightarrow K, B \rightarrow D$ and $B \rightarrow \pi$ form factors with, for comparison, a vector dominance, monopole model Eq. (31) and a lattice simulation [23]. Our results: continuous lines. Monopole: dotted lines. Lattice: data points. 